\title{
The EPA Conventional Reduced Risk Pesticide Program ${ }^{1}$
}

\author{
F.M. Fishel ${ }^{2}$
}

\section{Introduction}

The Food Quality Protection Act (FQPA) of 1996 initiated the U.S. Environmental Protection Agency's (EPA) Conventional Reduced Risk Pesticide Program. Its purpose is to expedite the review and registration process of conventional pesticides that pose less risk to human health and the environment than existing conventional alternatives. Riskier conventional alternatives are those pesticides EPA deems as having neurotoxic, carcinogenic, reproductive, and developmental toxicity, or groundwater contamination effects. It serves as a means to ensure that reduced risk pesticides enter the channels of trade and are available to growers as soon as possible. Reduced risk decisions are made at the use level. The program does not apply to biological or antimicrobial pesticides, which are handled through separate expediting processes.

\section{Advantages of Reduced Risk Pesticides}

Compared to existing conventional pesticides, reduced risk pesticides may provide a number of benefits:

- low impact on human health

- lower toxicity to nontarget organisms (e.g., birds, fish, plants)
- low potential for groundwater contamination

- low use rates

- low pest resistance potential

- compatibility with Integrated Pest Management (IPM) practices

\section{Criteria for Consideration}

EPA established an expedited review for manufacturers applying to register pesticides that may reasonably be expected to accomplish at least one of the following:

- reduce the risks of pesticides to human health

- reduce the risks of pesticides to nontarget organisms

- reduce the potential for contamination of groundwater, surface water, or other valued environmental resources

- broaden the adoption of IPM strategies, or make such strategies more available or more effective

\footnotetext{
1. This document is PI224, one of a series of the Agronomy Department, Florida Cooperative Extension Service, Institute of Food and Agricultural Sciences, University of Florida. Original publication date January 2010. Revised April 2013. Visit the EDIS website at http://edis.ifas.ufl.edu.

2. F.M. Fishel, professor, Agronomy Department, and director, Pesticide Information Office; Florida Cooperative Extension Service, Institute of Food and Agricultural Sciences, University of Florida, Gainesville, FL 32611.
} 


\section{Carbamate and Organophosphate Pesticides and Current Use Trends}

Carbamates and organophosphates (OPs) are a group of closely related pesticides used in agriculture and nonagricultural sites that affect functioning of the nervous system by targeting the cholinesterase system. A main concern with these insecticides is acute toxicity. Additionally, one member of the carbamates widely used in Florida, aldicarb, is strictly regulated largely because of groundwater contamination concerns. Carbamates and OPs are among EPA's first priority group of pesticides for review under the FQPA. EPA made alternatives to OP pesticides the first priority for review and regulatory decision-making. The conventional Reduced Risk Pesticide Program screens OP alternatives for this initiative. Table 1 provides a list of reduced risk and OP alternative pesticides currently registered for use in the United States. Some active ingredients listed in Table 1 are not registered for use in Florida.

EPA determines if a candidate is a potentially significant OP alternative by an approach that includes, but is not limited to, consideration of the following factors:

- The affected OPs collectively have a significant market share for the specified use pattern.

- Currently registered alternatives, if any exist, have constraints that prevented their widespread adoption as alternatives to the affected OPs, such as inferior efficacy or pest-resistance issues.

- The proposed reduced risk alternative appears to overcome many of the constraints of the alternatives.

The IR-4 (Interregional Research Project No. 4) program is involved in making sure that pesticides are registered for use on minor crops. Minor-use pesticides are those that, for a variety of reasons, produce relatively little revenue for their manufacturers; they may be registered for use with a seldom-seen pest or for a crop that is not grown by a large number of producers. However, in Florida's agricultural setting, minor crops include some high-revenue fruit, vegetable, and ornamental crops. Based on publicly available data from the California Department of Pesticide Regulation and the CropLife Foundation, a 2009 report by IR-4 indicated that from 1994 to 2006, OP use in the United States has shown an overall decline by approximately $50 \%$. During the same period, carbamate use declined $70 \%$.

A direct benefit of the reduction has been to the environmental load. The environmental load is the rate of application (lbs/acre) of chemicals to the environment. The reduced risk pesticides are generally used at significantly lower application rates than the conventional compounds they are replacing, which has the effect of decreasing the amount of chemical applied to the environment. The trend from 1994 to 2006 has shown a 45\% combined decrease in the environmental load for the carbamate and organophosphate insecticides.

Acute toxicity concerns have also been addressed with the increased number of reduced risk pesticides currently registered for use. Of the cholinesterase-inhibiting insecticides, $73 \%$ of these compounds most widely used in the United States fall into the highest toxicity class of EPA and none are in the safest class. By contrast, $64 \%$ of the reduced risk insecticides fall into the highest safety class, and the rest are in the next safest group III.

\section{Additional Information}

Fishel, F.M. 2011. Pesticide Toxicity Profile: Organophosphate Pesticides. PI-50. Gainesville: University of Florida Institute of Food and Agricultural Sciences. http://edis.ifas. ufl.edu/pi087.

Fishel, F.M. 2012. Pesticides and Cholinesterase. PI-221. Gainesville: University of Florida Institute of Food and Agricultural Sciences. http://edis.ifas.ufl.edu/pi221.

Fishel, F.M. 2013. Specifically Regulated Pesticides in Florida - Aldicarb. PI-74. Gainesville: University of Florida Institute of Food and Agricultural Sciences. http://edis.ifas.ufl.edu/ pil11.

Nesheim, O.N., F.M. Fishel, and M.A. Mossler. 2011. Toxicity of Pesticides. PI-13. Gainesville: University of Florida Institute of Food and Agricultural Sciences. http://edis.ifas. ufl.edu/pi008.

Olexa, M.T., and Z. Broome. 2011. Handbook of Florida Water Regulation: Food Quality Protection Act. FE589. Gainesville: University of Florida Institute of Food and Agricultural Sciences. http://edis.ifas.ufl.edu/fe589.

Viray, F.A., and R. Hollingworth. 2009. "The Use and Benefits of Reduced Risk Pesticides since the Passage of the Food Quality Protection Act." The IR-4 Project Newsletter Volume 40, Number 4. New Jersey Agricultural Experiment Station. Accessed March 2013. http://ir4.rutgers.edu/ Newsletter/vol40no4.pdf. 
Table 1. Reduced risk (RR)/OP alternative pesticides registered in the United States.

\begin{tabular}{|c|c|c|c|c|}
\hline Year & Pesticide $^{*}$ & Pesticide type & Site & $\begin{array}{l}\text { Reduced risk (RR)/OP } \\
\text { alternative }^{\dagger}\end{array}$ \\
\hline \multirow[t]{2}{*}{1994} & Hexaflumuron & Insecticide & Belowground bait station (termites) & $\mathrm{RR}$ \\
\hline & Methyl anthranilate & Repellent & Cherry, blueberry, grape, forestry & $\mathrm{RR}$ \\
\hline \multirow[t]{3}{*}{1995} & Flumiclorac-pentyl & Herbicide & Corn, soybean & RR \\
\hline & Tebufenozide & Insecticide & Walnut & $\mathrm{RR}$ \\
\hline & Hymexazol & Fungicide & Sugar beet (seed treatment) & RR \\
\hline \multirow[t]{3}{*}{1996} & Fludioxonil & Fungicide & Corn & RR \\
\hline & Imazapic & Herbicide & Peanut & $\mathrm{RR}$ \\
\hline & Mefenoxam & Fungicide & All metalaxyl uses & $\mathrm{RR}$ \\
\hline \multirow[t]{6}{*}{1997} & Azoxystrobin & Fungicide & Non-residential turf & RR \\
\hline & Spinosad & Insecticide & Cotton & RR \\
\hline & Alpha-metolachlor & Herbicide & All metolachlor uses & RR \\
\hline & Imazamox & Herbicide & Soybean & $\mathrm{RR}$ \\
\hline & Hexaflumuron & Insecticide & Aboveground bait station (termites) & RR \\
\hline & Azoxystrobin & Fungicide & $\begin{array}{l}\text { Grape, banana, peach, tomato, pecan, } \\
\text { peanut }\end{array}$ & RR \\
\hline \multirow[t]{7}{*}{1998} & Fludioxonil & Fungicide & $\begin{array}{l}\text { Potato and seed treatments (many } \\
\text { crops) }\end{array}$ & RR \\
\hline & Diflubenzuron & Insecticide & Belowground bait station (termites) & RR \\
\hline & Cyprodinil & Fungicide & Stone fruit & $\mathrm{RR}$ \\
\hline & Spinosad & Insecticide & $\begin{array}{l}\text { Almond, apple, citrus, brassica leafy } \\
\text { vegetables, fruiting vegetables, and } \\
\text { leafy vegetables }\end{array}$ & $\mathrm{RR}$ \\
\hline & Pyriproxyfen & Insecticide & Cotton & $\mathrm{RR}$ \\
\hline & Tebufenozide & Insecticide & Pecan & RR \\
\hline & Carfentrazone-ethyl & Herbicide & Wheat, corn & RR \\
\hline \multirow[t]{12}{*}{1999} & Azoxystrobin & Fungicide & $\begin{array}{l}\text { Turf (residential), almond, cucurbit } \\
\text { vegetables, rice, wheat, canola, } \\
\text { potato, stone fruit }\end{array}$ & $\mathrm{RR}$ \\
\hline & Diflufenzopyr & Herbicide & Corn & $\mathrm{RR}$ \\
\hline & Tebufenozide & Insecticide & $\begin{array}{l}\text { Leafy, brassica, and fruiting } \\
\text { vegetables, cranberry, forestry, } \\
\text { ornamentals, berry crop group, mint, } \\
\text { pome fruit, cotton, sugarcane, turnip, } \\
\text { canola }\end{array}$ & $\mathrm{RR} / \mathrm{OP}$ \\
\hline & Pyriproxyfen & Insecticide & Pome fruit, walnut & $\mathrm{RR} / \mathrm{OP}$ \\
\hline & Glyphosate & Herbicide & $\begin{array}{l}\text { Glyphosate-tolerant corn, canola, } \\
\text { sugar beet }\end{array}$ & $\mathrm{RR}$ \\
\hline & s-Dimethenamid & Herbicide & Corn, soybean, peanut & $\mathrm{RR}$ \\
\hline & Spinosad & Insecticide & $\begin{array}{l}\text { Sweet corn, cucurbit and legume } \\
\text { vegetables, stone fruit, cereal grains }\end{array}$ & $\mathrm{RR} / \mathrm{OP}$ \\
\hline & Fenhexamid & Fungicide & Grape, strawberry, ornamentals & $\mathrm{RR}$ \\
\hline & Bifenazate & Insecticide & Ornamentals & $\mathrm{RR} / \mathrm{OP}$ \\
\hline & Trifloxystrobin & Fungicide & $\begin{array}{l}\text { Pome fruit, grape, cucurbit vegetables, } \\
\text { peanut, turf, banana, ornamentals }\end{array}$ & $\mathrm{RR}$ \\
\hline & Fipronil & Insecticide & Outside home use (termites) & OP \\
\hline & Pymetrozine & Insecticide & $\begin{array}{l}\text { Tuberous and corm vegetables, } \\
\text { ornamentals, tobacco }\end{array}$ & $\mathrm{RR} / \mathrm{OP}$ \\
\hline
\end{tabular}




\begin{tabular}{|c|c|c|c|c|}
\hline Year & Pesticide ${ }^{*}$ & Pesticide type & Site & $\begin{array}{l}\text { Reduced risk (RR)/OP } \\
\text { alternative }^{\dagger}\end{array}$ \\
\hline \multirow[t]{15}{*}{2000} & Pyriproxyfen & Insecticide & Citrus, fruiting vegetables, tree nuts & $\mathrm{RR} / \mathrm{OP}$ \\
\hline & Tebufenozide & Insecticide & Ornamentals (residential), tree nuts & $\mathrm{RR} / \mathrm{OP}$ \\
\hline & Ecolyst & $\begin{array}{l}\text { Herbicide/ } \\
\text { Insecticide/Plant } \\
\text { growth regulator }\end{array}$ & Orange & $\mathrm{RR}$ \\
\hline & Spinosad & Insecticide & $\begin{array}{l}\text { Non-grass animal feed crop group, } \\
\text { grain amaranth, cilantro, grass, } \\
\text { buckwheat, rye, pistachio, oat, barley, } \\
\text { millet, apple, popcorn, ti leaves, } \\
\text { watercress, tropical fruit, teosinte, } \\
\text { turnip greens }\end{array}$ & $\mathrm{RR} / \mathrm{OP}$ \\
\hline & Fenhexamid & Fungicide & Almond, stone fruit & $\mathrm{RR}$ \\
\hline & Prohexadione calcium & $\begin{array}{l}\text { Herbicide/ } \\
\text { Plant growth regulator }\end{array}$ & Apple & $\mathrm{RR}$ \\
\hline & Methoxyfenozide & Insecticide & Cotton, pome fruit & $\mathrm{RR} / \mathrm{OP}$ \\
\hline & Trifloxystrobin & Fungicide & $\begin{array}{l}\text { Almond, fruiting vegetables, } \\
\text { hops, potato, sugar beet, wheat, } \\
\text { ornamentals }\end{array}$ & $\mathrm{RR}$ \\
\hline & Carfentrazone-ethyl & Herbicide & Cereal grains & RR \\
\hline & Buprofezin & Insecticide & Cucurbit vegetables, head lettuce & $\mathrm{RR} / \mathrm{OP}$ \\
\hline & Fenpyroximate & Insecticide & Ornamentals (greenhouse) & $\mathrm{RR} / \mathrm{OP}$ \\
\hline & Indoxacarb & Insecticide & $\begin{array}{l}\text { Cotton, fruiting and brassica leafy } \\
\text { vegetables, lettuce, sweet corn, pome } \\
\text { fruit }\end{array}$ & $\mathrm{RR} / \mathrm{OP}$ \\
\hline & Flucarbazone-sodium & Herbicide & Wheat & RR \\
\hline & Glyphosate & Herbicide & $\begin{array}{l}\text { Many: refer to http://www. } \\
\text { epa.gov/opprd001/workplan/ } \\
\text { completionsportrait.pdf }\end{array}$ & $\mathrm{RR}$ \\
\hline & Azoxystrobin & Fungicide & $\begin{array}{l}\text { Barley, onion, citrus, corn (field, sweet, } \\
\text { pop), cotton, leafy, root, and tuberous } \\
\text { vegetables, soybean }\end{array}$ & RR \\
\hline
\end{tabular}




\begin{tabular}{|c|c|c|c|c|}
\hline Year & Pesticide $^{*}$ & Pesticide type & Site & $\begin{array}{l}\text { Reduced risk (RR)/OP } \\
\text { alternative }^{\dagger}\end{array}$ \\
\hline \multirow[t]{19}{*}{2001} & Fipronil & Insecticide & $\begin{array}{l}\text { Home lawn, golf course, commercial } \\
\text { and recreational turf and sod farms } \\
\text { (fire ant), potting medium mixtures } \\
\text { (fire ant) }\end{array}$ & OP \\
\hline & Thiamethoxam & Insecticide & $\begin{array}{l}\text { Barley, canola, cotton, sorghum, } \\
\text { wheat (all seed treatment), cotton, } \\
\text { pome fruit, cucurbit, fruiting, } \\
\text { tuberous, and corm vegetables (all } \\
\text { foliar) }\end{array}$ & OP \\
\hline & Fludioxonil & Fungicide & Strawberry, bulb vegetables, turf & $\mathrm{RR}$ \\
\hline & \multirow[t]{2}{*}{ Pyriproxyfen } & \multirow[t]{2}{*}{ Insecticide } & Food handling establishments & $\mathrm{RR}$ \\
\hline & & & Pistachio & $\mathrm{RR} / \mathrm{OP}$ \\
\hline & Imidacloprid & Insecticide & Leaf petioles, citrus & OP \\
\hline & Zoxamide & Fungicide & Grape, cucurbit vegetables, tomato & $\mathrm{RR}$ \\
\hline & Prohexadione calcium & Plant growth regulator & Grass (grown for seed) & $\mathrm{RR}$ \\
\hline & Pyriproxyfen & Insecticide & Pistachio & $\mathrm{RR} / \mathrm{OP}$ \\
\hline & Mesotrione & Herbicide & Corn (field) & $\mathrm{RR}$ \\
\hline & Cyprodinil & Fungicide & $\begin{array}{l}\text { Onion (dry, bulb, and green), } \\
\text { strawberry }\end{array}$ & $\mathrm{RR}$ \\
\hline & Buprofezin & Insecticide & Almond, citrus, cotton, grape, tomato & $\mathrm{RR} / \mathrm{OP}$ \\
\hline & \multirow[t]{2}{*}{ Carfentrazone-ethyl } & \multirow[t]{2}{*}{ Herbicide } & Cotton (defoliant use) & OP \\
\hline & & & Turf & $\mathrm{RR}$ \\
\hline & Fluazinam & Fungicide & Peanut, potato & $\mathrm{RR}$ \\
\hline & zeta-Cypermethrin & Insecticide & $\begin{array}{l}\text { Alfalfa, corn (field, pop, sweet), head } \\
\text { and stem brassica vegetables, leafy } \\
\text { brassica greens, leafy vegetables, } \\
\text { onion (green), sugar beet, sugarcane, } \\
\text { rice }\end{array}$ & OP \\
\hline & Azoxystrobin & Fungicide & $\begin{array}{l}\text { Leafy brassica greens, blueberry, } \\
\text { eggplant, grass (grown for seed), } \\
\text { jackfruit, juneberry, lingonberry, } \\
\text { loquat, mint (spearmint, peppermint), } \\
\text { okra, pawpaw, pepper, persimmon, } \\
\text { salal, strawberry, tamarind, tropical } \\
\text { fruit, turnip (greens), watercress, wax } \\
\text { jambu, white sapote }\end{array}$ & $\mathrm{RR}$ \\
\hline & Novaluron & Insecticide & Ornamentals (indoors, non-food) & $\mathrm{RR}$ \\
\hline & Spinosad & Insecticide & $\begin{array}{l}\text { Artichoke (globe), asparagus, } \\
\text { bushberry, cranberry, foliage of } \\
\text { legume vegetables, garden beet } \\
\text { (root), juneberry, leaves of root and } \\
\text { tuber vegetables, lingonberry, okra, } \\
\text { pistachio, pome fruit, salal, strawberry, } \\
\text { sugar beet (root), tree nuts }\end{array}$ & $\mathrm{RR} / \mathrm{OP}$ \\
\hline
\end{tabular}




\begin{tabular}{|c|c|c|c|c|}
\hline Year & Pesticide $^{*}$ & Pesticide type & Site & $\begin{array}{l}\text { Reduced risk (RR)/OP } \\
\text { alternative }^{\dagger}\end{array}$ \\
\hline \multirow[t]{19}{*}{2002} & Chlorfenapyr & Insecticide & Post-construction control of termites & OP \\
\hline & Imazamox & Herbicide & $\begin{array}{l}\text { Alfalfa, canola, legume vegetables, } \\
\text { wheat }\end{array}$ & RR \\
\hline & \multirow[t]{2}{*}{ Pymetrozine } & \multirow[t]{2}{*}{ Insecticide } & $\begin{array}{l}\text { Cotton, leafy, head and stem brassica, } \\
\text { and leafy brassica vegetables, hops }\end{array}$ & $\mathrm{RR} / \mathrm{OP}$ \\
\hline & & & Pecans & OP \\
\hline & Bifenazate & Insecticide & $\begin{array}{l}\text { Cotton, grapes, hops, nectarine, } \\
\text { peach, plum, pome fruit, strawberry }\end{array}$ & $\mathrm{RR} / \mathrm{OP}$ \\
\hline & Acetamiprid & Insecticide & $\begin{array}{l}\text { Cotton, pome fruit, citrus, grapes, } \\
\text { brassica leafy, leafy (excl. brassica), } \\
\text { and fruiting vegetables, ornamentals }\end{array}$ & $\mathrm{RR} / \mathrm{OP}$ \\
\hline & Trifloxystrobin & Fungicide & $\begin{array}{l}\text { Citrus, corn (field, pop), pecan, rice, } \\
\text { stone fruit }\end{array}$ & RR \\
\hline & Cyhalofop-butyl & Herbicide & Rice & $\mathrm{RR}$ \\
\hline & Indoxacarb & Insecticide & Alfalfa, peanut, potato, soybean & $\mathrm{RR} / \mathrm{OP}$ \\
\hline & Fludioxonil & Fungicide & $\begin{array}{l}\text { Caneberry, pistachio, stone fruit, } \\
\text { watercress }\end{array}$ & RR \\
\hline & Pyriproxyfen & Insecticide & Stone fruit, blueberry, lychee, guava & $\mathrm{RR} / \mathrm{OP}$ \\
\hline & Imazethapyr & Herbicide & Rice & $\mathrm{RR}$ \\
\hline & Diflufenzopyr & Herbicide & Corn (pop, sweet), grass (forage, hay) & $\mathrm{RR}$ \\
\hline & Macalayea extract & Fungicide & Greenhouse ornamentals & $\mathrm{RR}$ \\
\hline & Azoxystrobin & Fungicide & Legume vegetables & RR \\
\hline & Methoxyfenozide & Insecticide & $\begin{array}{l}\text { Fruiting, leafy, and brassica leafy } \\
\text { vegetables, grapes, corn (field, sweet), } \\
\text { stone fruit, tree nuts }\end{array}$ & $\mathrm{RR} / \mathrm{OP}$ \\
\hline & Fenamidone & Fungicide & Lettuce & $\mathrm{RR}$ \\
\hline & Lambda-cyhalothrin & Insecticide & $\begin{array}{l}\text { Legume and fruiting vegetables, } \\
\text { sugarcane }\end{array}$ & RR (sugarcane)/OP (all) \\
\hline & Spinosad & Insecticide & $\begin{array}{l}\text { Berry group, fig, grape, herbs, peanut, } \\
\text { root and tuber vegetables }\end{array}$ & $\mathrm{RR} / \mathrm{OP}$ \\
\hline
\end{tabular}




\begin{tabular}{|c|c|c|c|c|}
\hline Year & Pesticide $^{*}$ & Pesticide type & Site & $\begin{array}{l}\text { Reduced risk (RR)/OP } \\
\text { alternative }^{\dagger}\end{array}$ \\
\hline \multirow[t]{22}{*}{2003} & Lambda-cyhalothrin & Insecticide & Termite barrier & $\mathrm{RR}$ \\
\hline & Pyriproxyfen & Insecticide & $\begin{array}{l}\text { Brassica leafy and cucurbit vegetables, } \\
\text { olive }\end{array}$ & $\mathrm{RR} / \mathrm{OP}$ \\
\hline & Cyprodinil & Fungicide & $\begin{array}{l}\text { Bushberry, caneberry, pistachio, } \\
\text { watercress, brassica leafy vegetables, } \\
\text { carrot, herbs, lychee fruits }\end{array}$ & $\mathrm{RR}$ \\
\hline & EH-2001 Rodenticide & Rodenticide & Richardson/Wyoming ground squirrel & $\mathrm{RR}$ \\
\hline & Mesotrione & Herbicide & Corn (pop) & $\mathrm{RR}$ \\
\hline & Noviflumuron & Insecticide & Aboveground bait station & $\mathrm{RR} / \mathrm{OP}$ \\
\hline & Pyriproxyfen & Insecticide & $\begin{array}{l}\text { Avocado fruits, fig, okra, sugar apple } \\
\text { fruits }\end{array}$ & $\mathrm{RR} / \mathrm{OP}$ \\
\hline & Clothianidin & Insecticide & Canola, corn (seed treatments) & OP \\
\hline & Methoxyfenozide & Insecticide & $\begin{array}{l}\text { Cranberry, cucurbits, okra, peas } \\
\text { (black-eyed, southern), turnip (greens) }\end{array}$ & $\mathrm{RR} / \mathrm{OP}$ \\
\hline & Azoxystrobin & Fungicide & $\begin{array}{l}\text { Artichoke (globe), asparagus, head } \\
\text { and stem brassica subgroup, herbs }\end{array}$ & $\mathrm{RR}$ \\
\hline & Emamectin benzoate & Insecticide & Cotton, fruiting vegetables, tobacco & OP \\
\hline & Buprofezin & Insecticide & $\begin{array}{l}\text { Bean (succulent), lychee fruits, } \\
\text { pistachio }\end{array}$ & $\mathrm{RR} / \mathrm{OP}$ \\
\hline & Boscalid & Fungicide & $\begin{array}{l}\text { Berries, bulb, fruiting, legume (root } \\
\text { except sugar beet, garden beet, } \\
\text { radish, turnip), tuberous and corm } \\
\text { vegetables, grape, lettuce (head, leaf), } \\
\text { peanut, stone fruit, strawberry, tree } \\
\text { nuts, turf }\end{array}$ & $\mathrm{RR}$ \\
\hline & Thiamethoxam & Insecticide & $\begin{array}{l}\text { Ornamentals, succulent beans (seed), } \\
\text { stone fruit, sunflower (seed) }\end{array}$ & OP \\
\hline & Trifloxystrobin & Fungicide & $\begin{array}{l}\text { Root vegetables leaf petioles (except } \\
\text { sugar beet) subgroup, except radish }\end{array}$ & $\mathrm{RR}$ \\
\hline & Flonicamid & Insecticide & Ornamentals (greenhouse) & OP \\
\hline & Acequinocyl & Insecticide & Ornamentals (greenhouse) & $\mathrm{RR}$ \\
\hline & Bifenazate & Insecticide & $\begin{array}{l}\text { Cucurbits, fruiting vegetables, mint, } \\
\text { pistachio, tomato (greenhouse), tree } \\
\text { nuts }\end{array}$ & $\mathrm{RR}$ \\
\hline & Fenhexamid & Fungicide & $\begin{array}{l}\text { Cucumber (greenhouse), fruiting } \\
\text { vegetables (except non-bell pepper), } \\
\text { kiwifruit, leafy green subgroup } \\
\text { (except spinach), stone fruit }\end{array}$ & $\mathrm{RR}$ \\
\hline & Etoxazole & Insecticide & Cotton, pome fruit, strawberry & $\mathrm{RR}$ \\
\hline & Quinoxyfen & Fungicide & Grape, hops, cherry & $\mathrm{RR}$ \\
\hline & Glufosinate-ammonium & Herbicide & Rice & $\mathrm{RR}$ \\
\hline
\end{tabular}




\begin{tabular}{|c|c|c|c|c|}
\hline Year & Pesticide $^{*}$ & Pesticide type & Site & $\begin{array}{l}\text { Reduced risk (RR)/OP } \\
\text { alternative }^{\dagger}\end{array}$ \\
\hline \multirow[t]{16}{*}{2004} & Fluroxypyr & Herbicide & Corn (field, sweet) & RR \\
\hline & Mesosulfuron-methyl & Herbicide & Wheat & RR \\
\hline & Gamma-cyhalothrin & Insecticide & $\begin{array}{l}\text { Alfalfa, brassica head and stem } \\
\text { subgroup, canola, corn (field, sweet), } \\
\text { cotton, fruiting and legume (edible- } \\
\text { podded) subgroup vegetables, garlic, } \\
\text { lettuce (head, leaf), tree nuts, onion } \\
\text { (dry bulb), pea and bean dry shelled } \\
\text { (except soybean) subgroup, pea and } \\
\text { bean succulent shelled subgroup, } \\
\text { peanut, pome fruit, rice, sorghum, } \\
\text { soybean, stone fruit, sugarcane, } \\
\text { sunflower, wheat }\end{array}$ & OP \\
\hline & Novaluron & Insecticide & Cotton, pome fruit & OP \\
\hline & Fenpyroximate & Insecticide & Cotton, grape, pome fruit & RR \\
\hline & Acequinocyl & Insecticide & $\begin{array}{l}\text { Strawberry, almond, citrus, pome fruit, } \\
\text { field ornamentals }\end{array}$ & RR \\
\hline & Lufenuron & Insecticide & Termite bait station & RR \\
\hline & Indoxacarb & Insecticide & Fire ant bait & $\mathrm{RR} / \mathrm{OP}$ \\
\hline & Pyrimethanil & Fungicide & $\begin{array}{l}\text { Almond, grape, onion (dry bulb, } \\
\text { green), pome and stone fruit, } \\
\text { strawberry, tomato, tuberous and } \\
\text { corm vegetables }\end{array}$ & RR \\
\hline & Dinotefuran & Insecticide & Leafy vegetables & $\mathrm{RR} / \mathrm{OP}$ \\
\hline & Penoxsulam & Herbicide & Rice & $\mathrm{RR}$ \\
\hline & Tebufenozide & Insecticide & $\begin{array}{l}\text { Citrus, grape, tuberous and corm } \\
\text { vegetables }\end{array}$ & $\mathrm{RR}$ \\
\hline & Fenamidone & Fungicide & $\begin{array}{l}\text { Cucurbit vegetables, onion (dry bulb, } \\
\text { green), potato, tomato }\end{array}$ & $\mathrm{RR}$ \\
\hline & Cyazofamid & Fungicide & Cucurbit vegetables, potato, tomato & RR \\
\hline & Bispyribac-sodium & Herbicide & Turf & $\mathrm{RR}$ \\
\hline & Deltamethrin & Insecticide & $\begin{array}{l}\text { Corn (field), cucurbit, fruiting, root and } \\
\text { tuber vegetables, onion (dry, bulb, } \\
\text { green), sorghum, tree nuts }\end{array}$ & OP \\
\hline
\end{tabular}




\begin{tabular}{|c|c|c|c|c|}
\hline Year & Pesticide $^{*}$ & Pesticide type & Site & $\begin{array}{l}\text { Reduced risk (RR)/OP } \\
\text { alternative }^{\dagger}\end{array}$ \\
\hline \multirow[t]{15}{*}{2005} & Fenamidone & Fungicide & Ornamentals & $\mathrm{RR}$ \\
\hline & Diflubenzuron & Insecticide & $\begin{array}{l}\text { Horse oral larvicide feed-through } \\
\text { treatment }\end{array}$ & $\mathrm{RR}$ \\
\hline & Dinotefuran & Insecticide & $\begin{array}{l}\text { Public health use, cotton, brassica } \\
\text { head and stem subgroup, cucurbit } \\
\text { and fruiting vegetables, grape, potato }\end{array}$ & $\mathrm{RR} / \mathrm{OP}$ \\
\hline & Clothianidin & Insecticide & Turf, ornamentals, pome fruit, tobacco & OP \\
\hline & Thiamethoxam & Insecticide & Mint & $\mathrm{OP}$ \\
\hline & Clofentezine & Insecticide & Grape & $\mathrm{RR}$ \\
\hline & Mesotrione & Herbicide & Corn (sweet) & $\mathrm{RR}$ \\
\hline & Buprofezin & Insecticide & $\begin{array}{l}\text { Avocado, guava, peach, pome fruit, } \\
\text { sugar apple }\end{array}$ & $\mathrm{RR} / \mathrm{OP}$ \\
\hline & Acetamiprid & Insecticide & Potato & $\mathrm{RR} / \mathrm{OP}$ \\
\hline & Spiromesifen & Insecticide & $\begin{array}{l}\text { Brassica leafy, fruiting, tuberous, and } \\
\text { corm vegetables, corn (field), cotton, } \\
\text { cucurbits, leafy greens, ornamentals, } \\
\text { strawberry }\end{array}$ & $\mathrm{RR}$ \\
\hline & Pymetrozine & Insecticide & Asparagus & $\mathrm{OP}$ \\
\hline & Etoxazole & Insecticide & Grape, tree nuts & $\mathrm{RR} / \mathrm{OP}$ \\
\hline & Pinoxaden & Herbicide & Barley, wheat & $\mathrm{RR}$ \\
\hline & Aminopyralid & Herbicide & $\begin{array}{l}\text { Range and pasture lands, rights-of- } \\
\text { way, roadsides, industrial vegetation } \\
\text { management }\end{array}$ & $\mathrm{RR}$ \\
\hline & Flonicamid & Insecticide & $\begin{array}{l}\text { Cotton, cucurbit and fruiting } \\
\text { vegetables, pome and stone fruit, } \\
\text { potato, nursery and landscape } \\
\text { ornamentals }\end{array}$ & $\mathrm{OP}$ \\
\hline \multirow[t]{11}{*}{2006} & Boscalid & Fungicide & Celery, spinach & $\mathrm{RR}$ \\
\hline & Flumiclorac-pentyl & Herbicide & Cotton defoliant use & $\mathrm{RR} / \mathrm{OP}$ \\
\hline & Spinosad & Insecticide & $\begin{array}{l}\text { Alfalfa, fruit fly bait, mint, onion } \\
\text { (green) }\end{array}$ & RR \\
\hline & Fenhexamid & Fungicide & $\begin{array}{l}\text { Ginseng, pear, cilantro, pepper (non- } \\
\text { bell), pomegranate }\end{array}$ & RR \\
\hline & Flonicamid & Insecticide & Head and stem brassica & $\mathrm{OP}$ \\
\hline & Trifloxystrobin & Fungicide & Barley, oats & $\mathrm{RR}$ \\
\hline & Azoxystrobin & Fungicide & Herbs, spices, safflower, sunflower & $\mathrm{RR}$ \\
\hline & Methoxyfenozide & Insecticide & Soybean & $\mathrm{RR} / \mathrm{OP}$ \\
\hline & Fenpyroximate & Insecticide & Citrus, hops, mint, pistachio, tree nuts & $\mathrm{RR}$ \\
\hline & Quinoxyfen & Fungicide & $\begin{array}{l}\text { Lettuce (head, leaf), melons, pepper } \\
\text { (bell, non-bell), strawberry }\end{array}$ & $\mathrm{RR}$ \\
\hline & Bifenazate & Insecticide & $\begin{array}{l}\text { Stone fruit, edible-podded pea, } \\
\text { tuberous and corm vegetables }\end{array}$ & $\mathrm{RR} / \mathrm{OP}$ \\
\hline
\end{tabular}




\begin{tabular}{|c|c|c|c|c|}
\hline Year & Pesticide $^{*}$ & Pesticide type & Site & $\begin{array}{l}\text { Reduced risk (RR)/OP } \\
\text { alternative }^{\dagger}\end{array}$ \\
\hline \multirow[t]{7}{*}{2007} & Fluthiacet-methyl & Herbicide & Cotton & $\mathrm{OP}$ \\
\hline & Spiromesifen & Insecticide & Tomato (greenhouse) & $\mathrm{RR}$ \\
\hline & Flazasulfuron & Fungicide & Turf & $\mathrm{RR}$ \\
\hline & Penoxsulam & Herbicide & Turf, aquatic use & $\mathrm{RR}$ \\
\hline & Indoxacarb & Insecticide & Grape & $\mathrm{RR}$ \\
\hline & Spinosad & Insecticide & Mosquito larvicide use & $\mathrm{RR}$ \\
\hline & Spinetoram & Insecticide & $\begin{array}{l}\text { Many: refer to http://www. } \\
\text { epa.gov/opprd001/workplan/ } \\
\text { completionsportrait.pdf }\end{array}$ & $\mathrm{RR}$ \\
\hline \multirow[t]{5}{*}{2008} & Mandipropamid & Fungicide & $\begin{array}{l}\text { Brassica leafy, bulb, cucurbit, fruiting, } \\
\text { tuberous and corm, and leafy } \\
\text { vegetables, grape }\end{array}$ & $\mathrm{RR}$ \\
\hline & Mesotrione & Herbicide & $\begin{array}{l}\text { Berry group, cranberry, flax, turf (sod } \\
\text { farm, golf courses) }\end{array}$ & $\mathrm{RR}$ \\
\hline & Chlorantraniliprole & Insecticide & $\begin{array}{l}\text { Cotton, grape, pome and stone fruit, } \\
\text { potato, turf, ornamentals, brassica } \\
\text { leafy, cucurbit, fruiting, and leafy } \\
\text { vegetables }\end{array}$ & $\mathrm{RR}$ \\
\hline & Spirotetramat & Insecticide & $\begin{array}{l}\text { Almond, citrus, grape, hops, onion } \\
\text { (bulb), brassica head and stem, } \\
\text { brassica leafy greens, cucurbits, } \\
\text { fruiting, leafy, and tuberous and corm } \\
\text { vegetables }\end{array}$ & $\mathrm{RR}$ \\
\hline & Etofenprox & Insecticide & Mosquito adulticide use & $\mathrm{RR}$ \\
\hline \multirow[t]{7}{*}{2009} & Etofenprox & Insecticide & Rice & $\mathrm{RR}$ \\
\hline & Mesotrione & Herbicide & Turf (commercial, residential) & $\mathrm{RR}$ \\
\hline & Spiromesifen & Insecticide & $\begin{array}{l}\text { Corn (pop, sweet), low-growing berry } \\
\text { group }\end{array}$ & $\mathrm{RR}$ \\
\hline & Penoxsulam & Herbicide & Grape, tree nuts & $\mathrm{RR}$ \\
\hline & Chlorantraniliprole & Insecticide & Tree nuts, pistachio & $\mathrm{RR}$ \\
\hline & Cyazofamid & Fungicide & $\begin{array}{l}\text { Fruiting vegetables (regional } \\
\text { tolerance), okra }\end{array}$ & $\mathrm{RR}$ \\
\hline & Saflufenacil & Herbicide & $\begin{array}{l}\text { Cereal grains, citrus, cotton, foliage } \\
\text { of legume vegetables, forage, fodder, } \\
\text { and straw of cereal grains, grape, } \\
\text { legume vegetables, pome and stone } \\
\text { fruit, sunflower, tree nuts }\end{array}$ & $\mathrm{RR}$ \\
\hline \multirow[t]{2}{*}{2010} & Dinotefuran & Insecticide & Brassica leafy greens, turnip (greens) & $\mathrm{RR}$ \\
\hline & Chlorantraniliprole & Insecticide & $\begin{array}{l}\text { Artichoke, asparagus, caneberry, } \\
\text { cacao, citrus, coffee, corn (field, sweet, } \\
\text { pop), fig, forage, fodder, and straw } \\
\text { of cereal grains, grass forage, fodder, } \\
\text { and hay, herbs and spices, hops, } \\
\text { legume vegetables (ex., soybean), } \\
\text { mint, non-grass animal feeds, oilseed } \\
\text { crops, okra, olive, peanut, persimmon, } \\
\text { pomegranate, prickly pear cactus, rice, } \\
\text { small vine-climbing fruits, strawberry, } \\
\text { sugar, cane, tea, tobacco, tropical } \\
\text { fruits, tuberous and corm vegetables, } \\
\text { termiticide use }\end{array}$ & RR \\
\hline
\end{tabular}




\begin{tabular}{|c|c|c|c|c|}
\hline Year & Pesticide ${ }^{*}$ & Pesticide type & Site & $\begin{array}{l}\text { Reduced risk (RR)/OP } \\
\text { alternative }^{\dagger}\end{array}$ \\
\hline & Tolfenpyrad & Insecticide & Ornamentals (greenhouse) & RR \\
\hline & Cyazofamid & Fungicide & $\begin{array}{l}\text { Brassica leafy vegetables, hops, } \\
\text { spinach, turnip (greens) }\end{array}$ & $\mathrm{RR}$ \\
\hline & Spiromesifen & Insecticide & Pea, dry & RR \\
\hline
\end{tabular}

